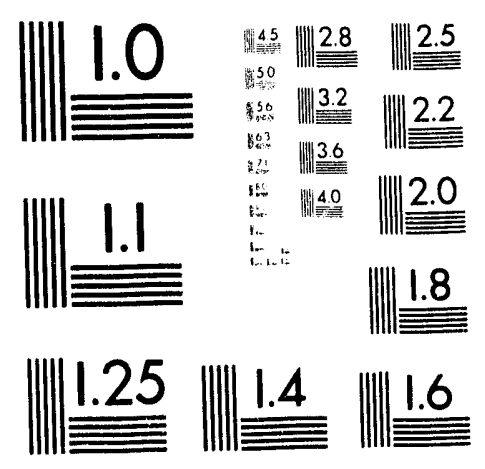



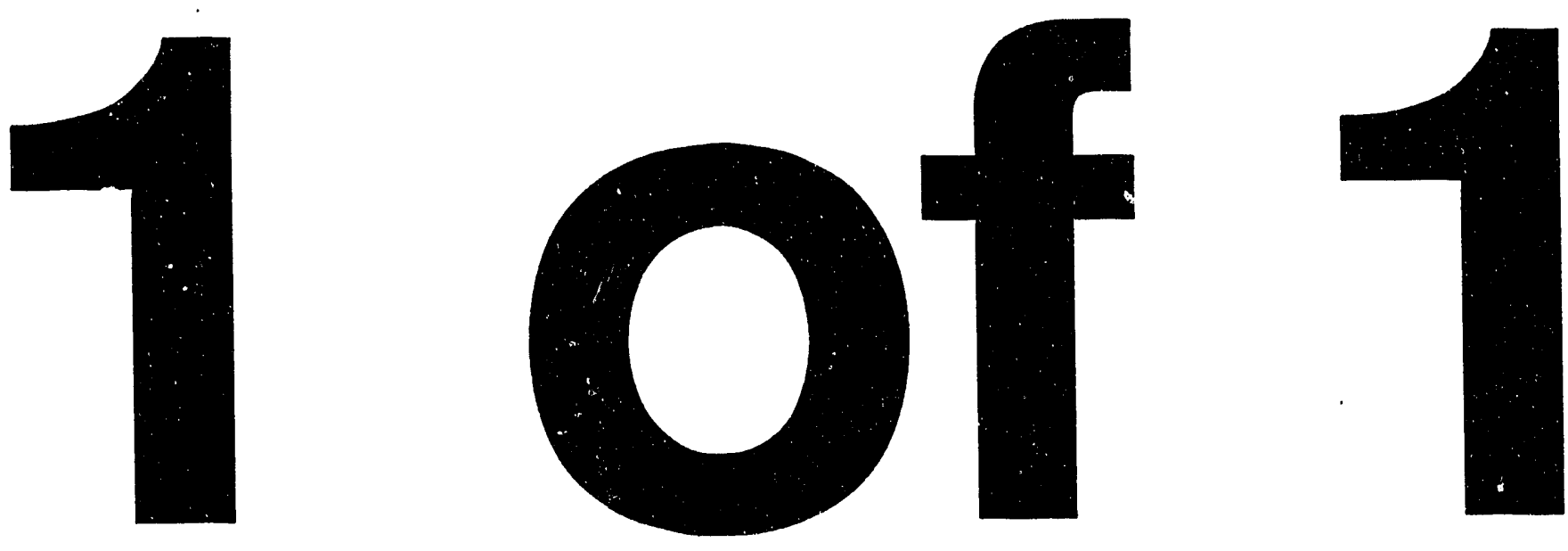
UCRL-JC-113895

PREPRINT

\title{
Microscopic and Macroscopic Dynamics
}

\author{
Wm. Hoover \\ C.G. Hoover \\ A.J. DeGroot \\ T.G. Pierce
}

This paper was prepared for submittal to the

Second International ACPC Conference on Parallel Computing

October 4-8, 1993

Vienna, Austria

June 1993

This is a preprint of a paper intended for publication in a journal or proceedings. Since changes may be made before publication, this preprint is made arailable with the understanding that it will not be cited or reproduced without the permission of the author. 


\section{DISCLAIMER}

This document was prepared as an account of work sponsored by an agemcy of the United States Government. Neither the United States Government nor the University of California nor any of their employees, makes any warranty, express or implied, or assumes any legal liability or responsihility for the accu racy, completeness, or usefulness of any information, apparat us, product, or process disdoxed, or represents that its use would not infringe privately ouned rights. Reference herein to any specific commercial products, process, or service by trade name, trademark, manufacturer, or otherwise, dexes nox necessarily constitute or imply its endorsement, recommendation, or favoring by the United States Govemment or the Lniversity of California. The views and opinions of authors expressed herein do not inecessarily state or renect those of the United States Govenument or the Univensity of Califomia, and shall not be used for advertising or product endorsement purposes. 


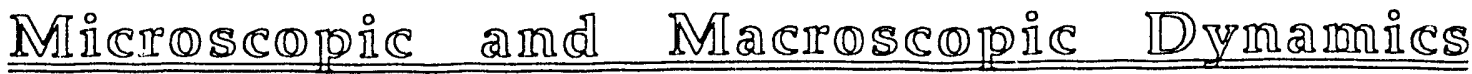

\section{Wm. G. Hoover, C. G. Hoover, A. J. De Groot, and T. G. Pierce}

Department of Applied Science, University of California at Davis/Livermore, and Lawrence Livermore National Laboratory, Livermore, California

Post Office Box 808, L-794, 94551-808, United States of America.

MANUSCRIPT COMPleted 1 JUNe 1993

\section{Abstract:}

Atomistic Molecular Dynamics and Lagrangian Continuum Mechanics can be very similarly adapted to massively-parallel computers. Millions of degrees of freedom can be treated. The two complementary approaches, microscopic and macroscopic, are being applied to increasingly realistic flows of fluids and solids. The two approaches can also be combined in a hybrid simulation scheme. Hybrids combine the fundamental constitutive advantage of atoms with the size advantage of the continuum picture.

\section{Introduction}

The computer revolution is rapidly progressing from millions of degrees of freedom to billions, and from gigaflops to teraflops. The impact of this ever-faster ever-cheaper computational power on physics and materials science is both exciting and unpredictable. Certainly we will have access to more realism and understanding, and to more detail in simulation. The consequences strain the imagination.

These gains are being achieved through parallelism in computation, the subject of this Conference. Parallel computers do best with problems which can be partitioned into weakly-interacting parts. Nodal descriptions of these parts are then assigned to individual processors. With more programming effort, the assignment of parts to processors can be made dynamically, redistributing coordinates, velocities, and energies as the nodes move. Again, with more effort, "Load Balancing" can be achieved by transferring work from busy processors to their less-busy neighbors. Even in the simplest case it is necessary to communicate at least some positions and velocities between neighboring processors, so as to calculate the accelerations. The time involved in such "Message-Passing" 
needs to be small compared to the simulation time steps. At the moment, the hardware limits imposed on this parallel simulation appruach are changing much more rapidly than the software can follow. As a result, it is a frustrating task to remain at the state of the art. It is much simpler to use the best readily-available commercial equipment.

With the help of Tony De Groot, who is in the process of building yet another massively-parallel machine, this one with 256 transputers, and my wife, Carol Hoover, who has been working on the 512-node CM5 Connection Machine at Minnesota for a year, I am in the fortunate position of seeing the best in both the home-built and commercial worlds of computer hardware. Tim Pierce, a student in our Department, who is also a fulltime programmer at the National Energy Research Supercomputer Center, has provided enthusiastic help and insight in implementing and visualizing new approaches to physical problems. My talk represents the recent efforts of all these colleagues, and so it is typical, though on a small scale, of the trend away from individual efforts and toward cooperating research groups.

The headlong advances in hardware are a cliché. But caution is desirable because more is not necessarily better. Atomistic force models proliferate and undergo ad hoc adjustment. Continuum "Failure Models" for simulations of elastic-plastic flow and fracture are a small industry. It is difficult, in both these typical cases, to have confidence in the predictive ability of the models. They inspire confidence only when used as interpolation devices: Extrapolation to new problems is risky.

Computers need to be used wisely. Cost-effective projects are likely to be neither so grandiose nor so predictable in their outcomes as are the socalled "Grand Challenges" - the genome, weather, and proton-mass problems. Lorenz' "Butterfly-Effect" weather model, Feigenbaum's logistic map studies, and Mandelbrot's complex-plane version of that map, have all had impacts completely out of proportion to the small-scale calculations leading to their discoveries. As computing machines evolve, their enhanced capacity and speed can be used for a more comprehensive treatment of flow and failure properties in which strengths are exploited and weaknesses avoided. 
2. Equations of Motion

Dynamics means motion. Newton's Second Law of motion can be applied equally well to individual atomistic masses or to the lumped continuum mass densities associated with individual nodes. In either case, the simplest way to generate approximate solutions of the equations of motion is to iterate Stoermer's "leapfrog" recipe through a series of timesteps separated by intervals of length $\mathrm{dt}$ :

$$
\left\{\left[q_{t+d t}-2 q_{t}+q_{t-d t}\right]=a_{t}(d t)^{2}\right\} .
$$

1

Here past, present, and future are the three times $\{t-d t, t, t+d t\}$. Future coordinate sets $\left\{q_{t+d t}\right\}$ can be calculated from the present and past ones, $\left\{q_{t}-d_{t}\right\}$ and $\left\{q_{t}\right\}$, by using present accelerations $\left\{a_{t}\right\}$. It is straightforward to generalize Stoermer's algorithm to include the velocity-dependent driving and constraint forces required in nonequilibrium simulations.

Stoermer's algorithm has several nice features. It is patently timereversible. Yoshida showed that there is a hidden and significant Hamiltonian basis underlying this simple reversible algorithm. The finitedifference solutions of Stoermer's equations, using forces from a Hamiltonian $\mathrm{H}$, trace out a sequence of phase points $\{\mathrm{q}, \mathrm{p}\}$. These same phase points lie on the exact continuous trajectory generated by a slightlydifferent perturbed Hamiltonian, $\mathrm{H}+\Delta \mathrm{H}$, where $\Delta \mathrm{H}$ is linear in the timestep dt. This Hamiltonian basis for Stoermer's algorithm accounts for its excellent stability properties.

In addition, for either atomistic or continuum simulations, the Stoermer leapfrog algorithm minimizes storage requirements. In continuum mechanics the largest possible timestep is typically used, so as to minimize spurious numerical diffusion. In atomistic mechanics a smaller, more conservative, choice is typical. If more thorough, or more speculative, investigations require it, even higher accuracy can be obtained from fourth-order integrators.

In the continuum case the degrees of freedom at locations $\{r\}$ have associated with them all the variables considered by molecular dynamics: density (or mass), velocity, and energy. But in addition the pressure tensor and heat flux vector must be known in order to evaluate the time evolution of a continuum system. 


\section{Work, Heat, and Boundary Conditions}

In the atomistic case the flows of momentum and energy are local mechanical variables. By measuring these, relative to the local stream velocity, the pressure tensor and heat flux vector can be identified. Thus, the dynamical aspect of the First Law of Thermodynamics, usually written $\mathrm{dE} \equiv \mathrm{dQ}-\mathrm{dW}$, can be made more-explicit:

$$
\mathrm{dE} / \mathrm{dt}=\mathrm{dQ}(\{\delta \mathrm{p}\}) / \mathrm{dt}-\mathrm{dW}(\{\delta \mathrm{q}\}) / \mathrm{dt} \text {. }
$$

As indicated here, the two types of power associated with (i) extracting heat $Q$ and (ii) doing work $W$ involve momentum and coordinate changes, respectively. About ten years ago the thermal part of thermodynamic energy flows was introduced into the differential equations of nonequilibrium molecular dynamics in a novel way. This thermostatted molecular dynamics incorporated frictional constraint forces,

$$
\begin{gathered}
\left\{\mathrm{F}_{\text {CONSTRAINT }}=-\zeta \mathrm{p}\right\} ; \\
\zeta_{\text {GAUSS }} \equiv-\mathrm{d} \Phi / \mathrm{dt} /(2 \mathrm{~K}) \text { or } \zeta_{\text {NOSÉ-HOOVER }} \equiv \int\left[\left(\mathrm{K} / \mathrm{K}_{\mathrm{O}}\right)-1\right] \mathrm{dt} / \tau^{2} .
\end{gathered}
$$

$\Phi(\{q\})$ and $K(\{p\})$ are the potential and kinetic energies. The friction coefficient(s) $\{\zeta\}$ obey one of the two feedback equations ("differential control", based on Gauss' Principle of Least Constraint, or Nosé-Hoover "integral control") and thereby control the temperature(s) of selected degrees of freedom. Similar feedback ideas could be used to stabilize the zero-point energies associated with individual molecular degrees of freedom, so as better to describe quantum systems.

The external heat sources or sinks represented by either of these reversible friction coefficients $\{\zeta\}$ undergo entropy changes, $\mathrm{dS}_{\text {EXTERNAL }} / \mathrm{dt}$ $=\mathrm{d}(\mathrm{Q} / \mathrm{T}) / \mathrm{dt}$, where $\mathrm{T}$ is the temperature associated with a particular source or sink, and the sum of these changes can be proved to satisfy the Second Law of Thermodynamics. Through either the Gauss or the NoséHoover feedback equations of motion, the time-averaged values of the friction coefficients $\{\langle\zeta\rangle\}$ can also be directly related to the spectrum of Lyapunov exponents $\{\lambda\}$. These relationships are: 


$$
<\mathrm{dS}_{\text {EXTERNAL }} / \mathrm{dt}>\equiv<\Sigma \zeta>\equiv<-\sum \lambda>\geq 0
$$

where the equals sign corresponds to the case where the net heat transfer vanishes. Though the individual friction coefficients fluctuate, and can be either positive or negative, the time-reversible microscopic equations of motion containing them have stable long-time solutions only in the case that the time-averaged friction coefficient sum is non-negative and the Lyapunov-exponent sum is negative. This inequality is the mechanical form of the Second Law of Thermodynamics. In geometrical terms the inequality states that, over time, phase-space volumes must shrink. Longterm growth, in a stationary state, would mean illegal instability.

The signatures of nonequilibrium states, either stationary or timeperiodic, are the multifractal strange attractors which they generate in phase space. Figure 1 shows such objects for five small few-body systems.
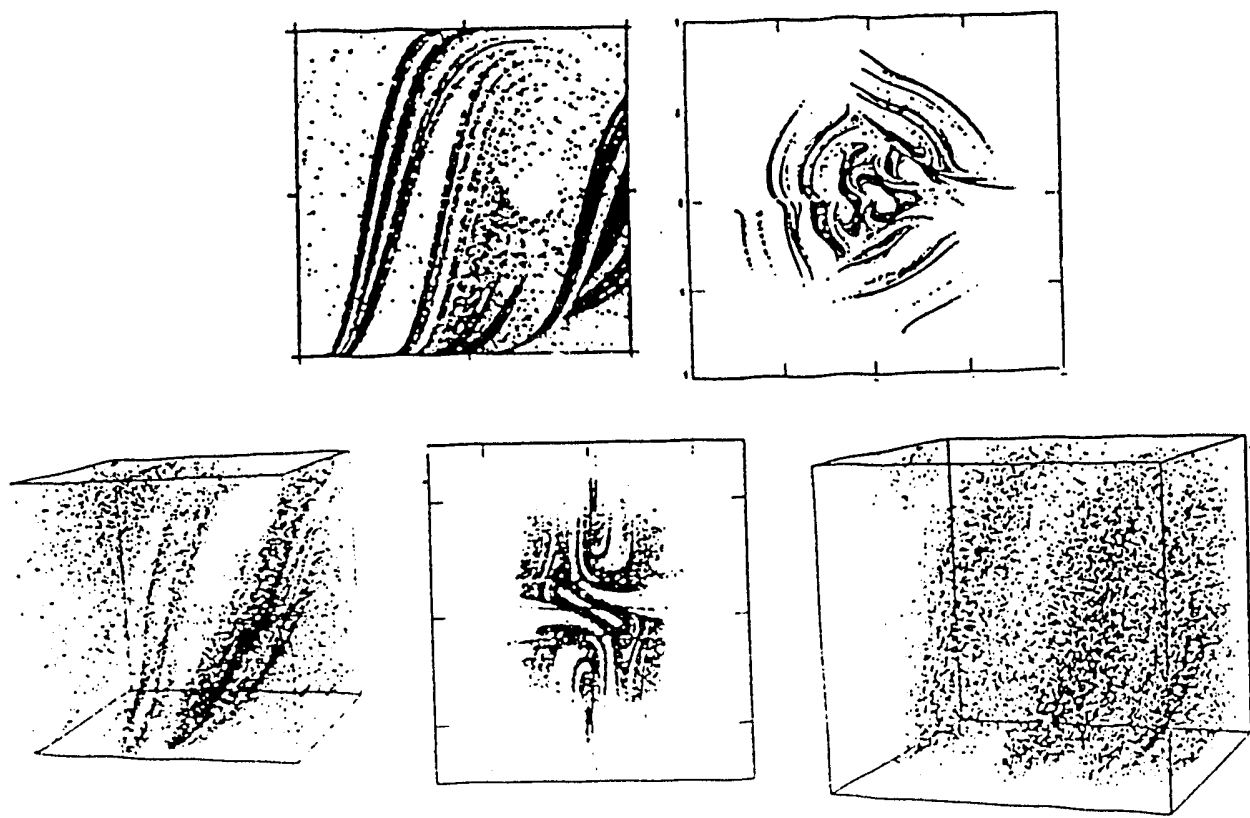

Figure 1. Two-and Three-Dimensional Poincaré sections of multifractal strange attractors with $\left\langle\sum \zeta\right\rangle \equiv\left\langle-\sum \lambda\right\rangle \geq 0$. These time-reversible distributions were all generated with time-reversible nonequilibrium equations of motion. For detailed references, see "Nonequilibrium Molecular Dynamics: the First 25 Years", Physica A 194, 450 (1993). 
It is still an unsolved problem how to describe the topological "lumpiness" of these objects. For larger many-body systems a geometric description of phase-space attractors is thoroughly hopeless. Instead, a simpler time-averaged description of the local rates of dissipation can be expressed in terms of the Lyapunov spectrum. The spectrum of Lyapunov exponents quantifies the direction-dependent stability of the underlying phase space flow. Each exponent describes the time-averaged rate of growth, or decay, of one of the principal axes of a comoving and corotating infinitestimal phase-space hyperellipsoid.

Because dissipation always corresponds to overall decay, the sum of the Lyapunov exponents is necessarily negative. Thus a comoving phasespace volume must shrink, and eventually vanish, as time goes on. On the other hand, the steady-state or time-periodic strange attractor, to which the motion is restricted once transients have decayed, must be stationary. The apparent paradox of "stationary shrinkage" can be resolved by noting that the zero-volume attractor has a reduced dimension [the "information dimension"]. Kaplan and Yorke suggested estimating this attractor dimension by finding the number of Lyapunov exponents in the spectrum required for their sum to vanish [corresponding to a topological object which neither grows nor shrinks]. Information dimensions have been estimated for nonequilibrium systems of up to a few hundred particles.

Today, just as in the early days of simulation following the Second World War, a 100-hour computer calculation represents a reasonable upper limit on one's attention span. In such a calculation today we can calculate only a few hundred Lyapunov exponents. A complete spectrum of $N$ exponents requires following $N$ additional trajectories, each described by $N$ ordinary differential equations of motion. The work involved typically varies (at least) as the cube of the number of phasespace coordinates N. My work with Harald Posch on systems with up to a few hundred degrees of freedom established that losses in dimension (embedding dimension less information dimension) can be substantial. The problem of characterizing this loss is one which will become much simpler with the next generation of parallel computers.

In the continuum case the proper treatment of material boundaries is less natural than in the atomistic case. Surface energy, essential to understanding failure, is most often ignored in numerical continuum treatments. The usual finite-element or finite-difference treatments rely 
on spatial grids, either "Eulerian" grids, fixed in space, or comoving "Lagrangian" grids, fixed in the material and following the flow.

To simplify intercomparisons between microscopic and macroscopic simulations, and to facilitate hybrid simulations, we have chosen here to concentrate on a "smoothed" or "smeared-out" free-Lagrange form of continuum mechanics called "Smoothed-Particle Hydrodynamics". This approach, invented by Lucy and Monaghan about 20 years ago, is nearly isomorphic to molecular dynamics, and so provides a natural extension of that microscopic approach. The equations of motion in smoothed-particle hydrodynamics incorporate accelerations depending upon the particle stress tensors $\{\sigma\}$ and on the gradients of normalized weighting functions $\left\{w\left(r_{i j}\right)\right\}$, which represent the mass distribution in the vicinity of each particle. We will describe this approach in more detail in Section 5.

\section{Molecular Dynamics}

In atomistic simulations, the goal of realism, quantitative agreement with experiment, remains elusive. After all, there is no practical approach to a nonequilibrium quantum many-body problem. But the still-worthy goal of understanding the mechanisms underlying classical nonequilibrium processes is now firmly within our grasp. Simulations of flows in channels with rvalls, of inelastic collisions between large bodies, of plastic flow with rapid deformation, and of shock deformation are becoming commonplace.

Vith CRAY1-speed work stations, simulations involving 10,000 atoms are routinely feasible. In the 40 -hour length of what most people consider a "long calculation" such a system can be followed through several sound traversal times. By linking together 1000 fast processors there is no difficulty in treating several million atoms, for a few specimen simulations. The Los Alamos CM5 "Connection Machine" has recently been used to provide timing tests for systems of as many as 108 individual atoms.

Figures 2 and 3 illustrate the simulated plastic deformation of solid silicon. The workpiece was originally a perfect cube, containing 373,248 silicon atoms. It was deformed by pressing a tetrahedral indentor into the workpiece. Though the microscopic details of the deformation are complicated, the energy relationships for the deforming silicon turn out to be relatively simple, justifying both the microscopic mechanistic approach and the macroscopic thermodynamic description of the results. 


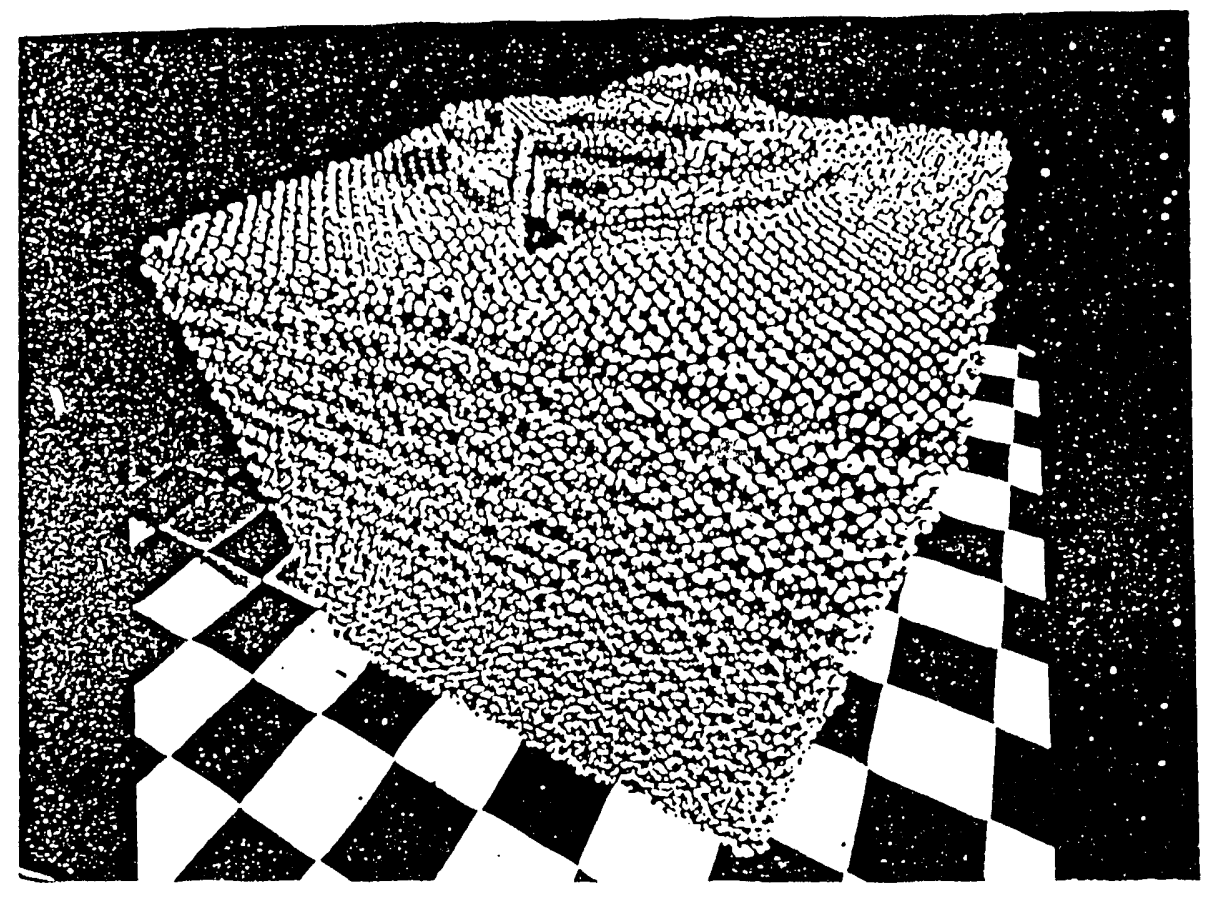

Figure 2. Final configuration of 373,248 silicon atoms after indentation. The temperature, maintained by a single Nosé-Hoover thermostat, is $15 \%$ of melting. The indentation velocity is about $1 / 3$ the sound velocity.
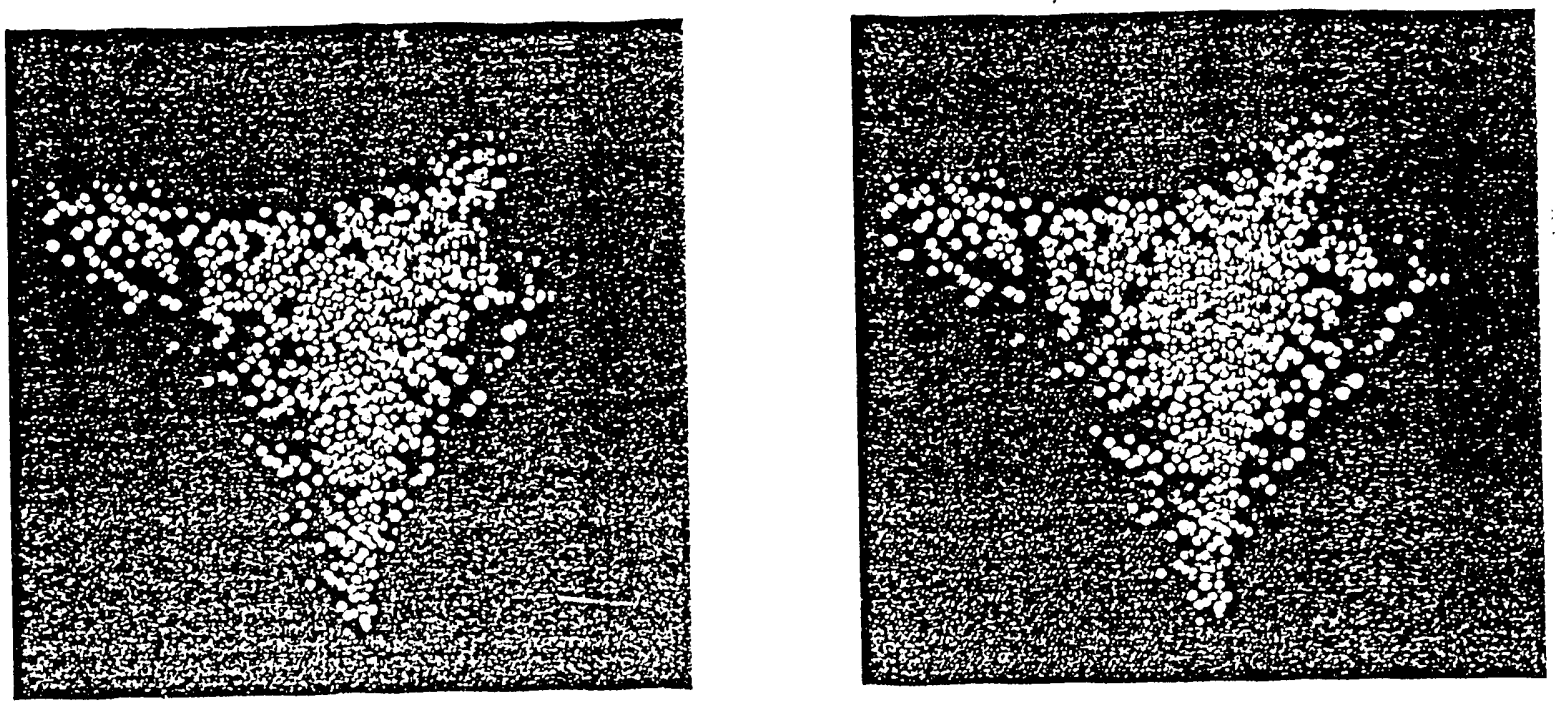

Figure 3. The initial positions of those atoms which interact with the indentor in Figure 2 are shown as a stereo pair. The images have been reversed, so that they need to be viewed with crossed eyes. 


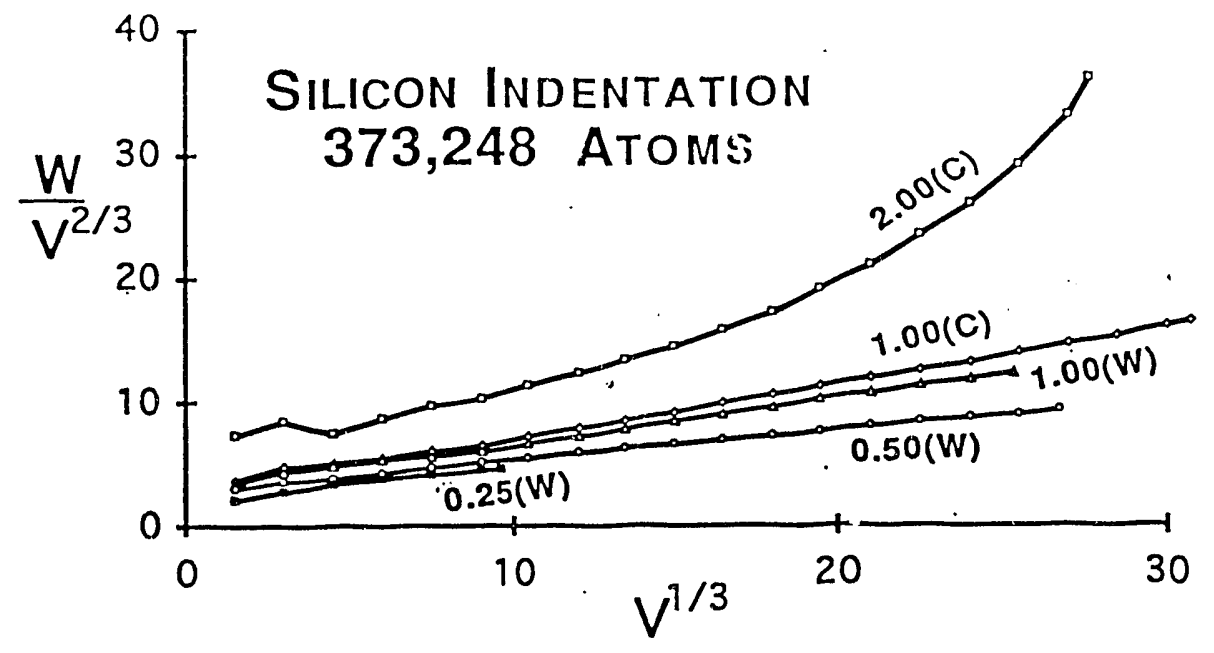

Figure 4. Variation of the work of indentation $\mathrm{W}$ with the tetrahedral indentation volume $\mathrm{V}$ for crystals of 373,248 silicon atoms. The energy, length, and mass units are 50 kilocalories/mole, 0.21 nanometers, and the mass of a silicon atom, respectively. The intercept is proportional to surface energy, while the slope is proportional to the plastic yield strength. $C=$ Cold and $W=W$ arm correspond to temperatures of about $15 \%$ and $45 \%$ of the melting temperature. The smooth-indentor speeds, with unity corresponding to 2.7 kilometers/second, are given.

In Figure 4 the macroscopic work of deformation $W$ is displayed as a function of indentation volume $\mathrm{V}$, so as to separate the bulk and surface contributions to the energy. For a tetrahedral indentor of height $h$, the volume of indentation $\mathrm{V}$ is $(31 / 2 / 8) \mathrm{h}^{3}$. We have separated the surface contributions $\left(\propto h^{2}\right)$ from the bulk contributions $\left(\propto h^{3}\right)$ by plotting $W / V^{2 / 3}$ as a function of $V^{1 / 3}$. The intercept then provides a surface energy estimate while the slope provides the yield strength. Our work on a variety of such systems shorvs that slowing the simulated indentation rate, to about one-tenth the sound speed, and increasing the workpiece size, to 10,000 atoms, provides very reasonable estimates of the quasistatic largecrystal yield strength, without much further sensitivity to speed or size.

Reproducing the rate- and temperature-dependence of the shapes of indentation pits is a challenging goal for continuum mechanics. To make headway on this problem with conventional continuum mechanics requires that a failure criterion be specified. The criterion allows computational nodes to divide, introducing new free surfaces within the material. 
With the use of work stations it is possible to analyze such nonequilibrium atomistic flows for information required for complementary continuum simulations. Where do the new surfaces come from in indentation? This question can be answered by coloring particles in the indented crystal according io their depth, but at positions corresponding to the undeformed crystal. Figure 3 shows those atoms destined to become surface atoms dirring the course of the indentation process. Detailed knowledge of this kird is vital to constructing faithful continuum models for failure.

\section{Smoothed Particle Hydrodynamics}

Smoothed particle hydrodynamics converts the partial differential equations of continuum mechanics into crdinary differential equations for particles. The resulting particle motion equations have nearly the same form as the molecular equations. The "particles" or nodes, each with mass $\mathrm{m}$, can be imagined to be $\ddot{c}$ istributed ("smoothed") over space, with a weighting function $\mathrm{w}$. The smoothed-particle equation of motion for the ith particle is a sum of pair interactions with nearby particles $\{j\}$ :

$$
\left\{\left(r_{t+d t}-2 r_{t}+r_{t-d t}\right)_{i}=m(d t) 2 \sum \nabla_{i} w_{i j}\left[\left(\sigma / \rho^{2}\right)_{i}+\left(\sigma / \rho^{2}\right)_{j}\right]\right\},
$$

where $\sigma$ and $\rho$ are local values of the stress tensor and mass density, and where the pairwise-additive weighting function $w\left(r_{i j}\right)$ is normalized and short-ranged. A simple choice for $w$, a caricature of a Gaussian weighting function, but with two vanishing derivatives at a maximum cutoff radius (here 1, for simplicity) is Lucy's:

$$
w(r) \propto(1+3 r)(1-r)^{3}, \text { for } r<1 .
$$

In validating the smoothed-particle approach it is natural to begin by checking that the linear hydrodynamic laws are satisfied. We have first to establish that shear flow and heat flow are correctly treated by the model. We did so by carrying out simple simulations of the types shown in Figure 5. The top and bottom boundaries are defined by constrained rows of smoothed particles, with fixed temperature and velocity. If the density of these particles is sufficiently high, the resulting barriers resemble Ashurst's 
"fluid wall" boundaries. The boundaries act as sources and sinks of momentum and energy, while preventing an outflux of mass. To prevent the occasional escape of bulk particles through the boundary, a Maxwell Demon reflects the normal velocity of any particle reaching the boundary. We built our confidence in the smoothed-particle method by verifying that the viscous shear stress and linear heat flux expected between walls of different temperatures and velocities were correctly reproduced, with statistical uncertainties of no more than a few percent.

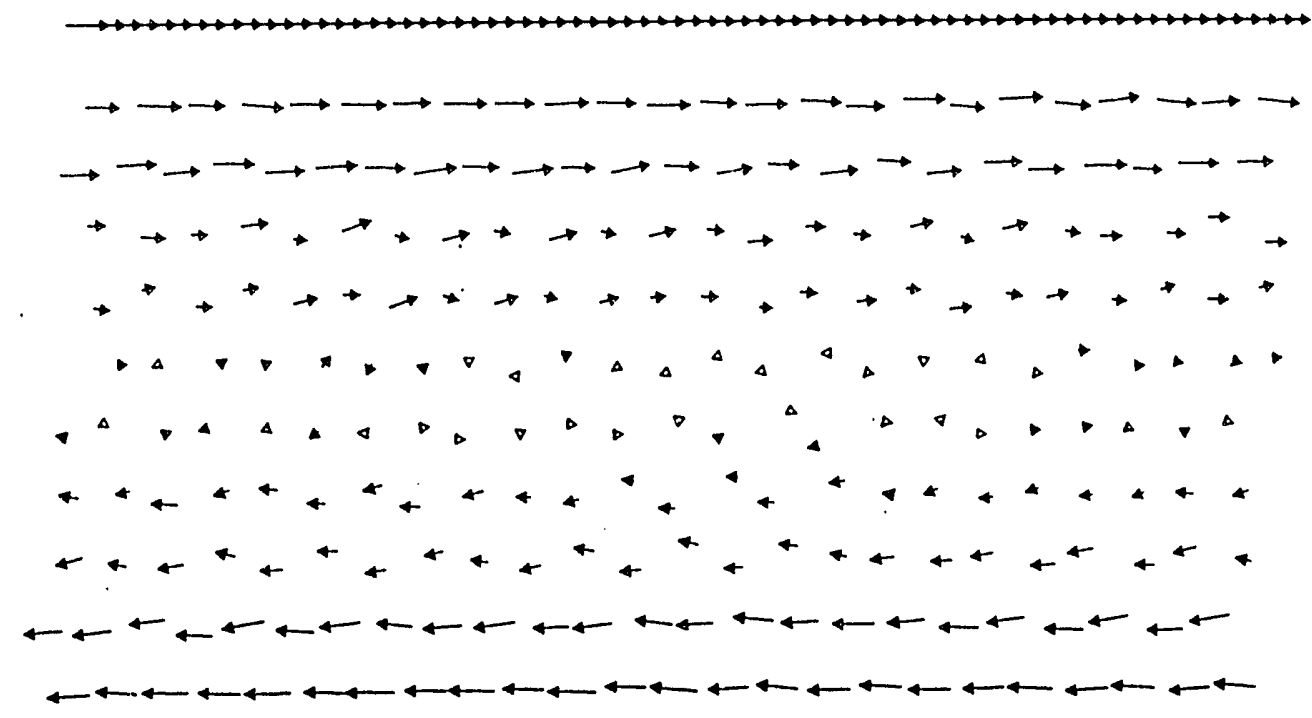

Figure 5.

Reservoirs, with temperature and average velocity fixed, are modelled by the constant-velocity constant-temperature smoothed particles.

Most applications of smoothed-particle hydrodynamics have been astrophysical. These are hard to validate because no independent trustworthy solutions are available. Because we wish to simulate relatively simple laboratory flows of fluids and solids we have chosen to investigate a simpler fluid flow problem, the Rayleigh-Bénard instability. This problem is two-dimensional, which simplifies visualization, and has previously been studied both with molecular dynamics and with conventional continuum methods. The three-equation caricature of this problem generates Lorenz' familiar "butterfly" attractor. Note that the smoothed-particle equations of motion reduce exactly to those of molecular dynamics [with pair potential $w(r)$ ] in the special case that the stress tensor is replaced by a hydrostatic stress corresponding to a twodimensional isentropic id aal gas with $\sigma \propto-\rho^{2}$. 
The nonlinear Payleigh-Bénard problem combines all three kinds of hydrodynamic flows: mass, momentum, and energy. In Rayleigh-Bénard flow a compressible fluid, in a gravitational field, is simultaneously heated from below and cooled from above. Two of the many earlier approaches to this problem, one atomistic and the other continuum, are shown in Figures 6 and 7. Snapshots from Rapaport's atomistic Rayleigh-Bénard simulation are shown in Figure 6. The system, hot on the bottom and cold on the top, transports heat with convective rolls. Rapaport's side walls are insulating. Figure 7 is a typical snapshot from Goldhirsch, Pelz, and Orszag's continuum simulation of Rayleigh-Bénard flow, based on a different boundary condition, with the temperature of the side walls varying linearly from the top to the bottom temperature.

In preparation for hybrid simulations combining the atomistic and continuum approaches, we have simulated this unstable Rayleigh-Bénard flow too, using a variety of boundary conditions. The constitutive model illustrated here is an ideal gas (with constant heat conductivity and shear viscosity). See Figures 8 and 9 for some or our sample results, not far from the 776-particle threshhold for convective transport. Our comparisons so far suggest that our particle description of the flow is inherently more chaotic than are the more-traditional Eulerian approaches.

In our first efforts to replicate Rayleigh- Bénard flow we used Lucy's weight function, and noticed an unphysical tendency for the smoothed particles to clump together. The model's bland acceptance of high-density collapse occurs because the gradient of the weighting function, which contributes to the repulsive force between the particles, vanishes (linearly) as $\mathrm{r}$ approaches zero. This tendency toward collapse can be eliminated by using an even simpler form for the weighting function (with a repulsive cusp at the origin): $\mathrm{w}(\mathrm{r}) \propto(1-\mathrm{r})^{3}$. The range of the weighting function is important too. In two dimensions our results for simulations using several hundred smoothed particles suggest that an interaction range covering about a dozen particles is optimum. 


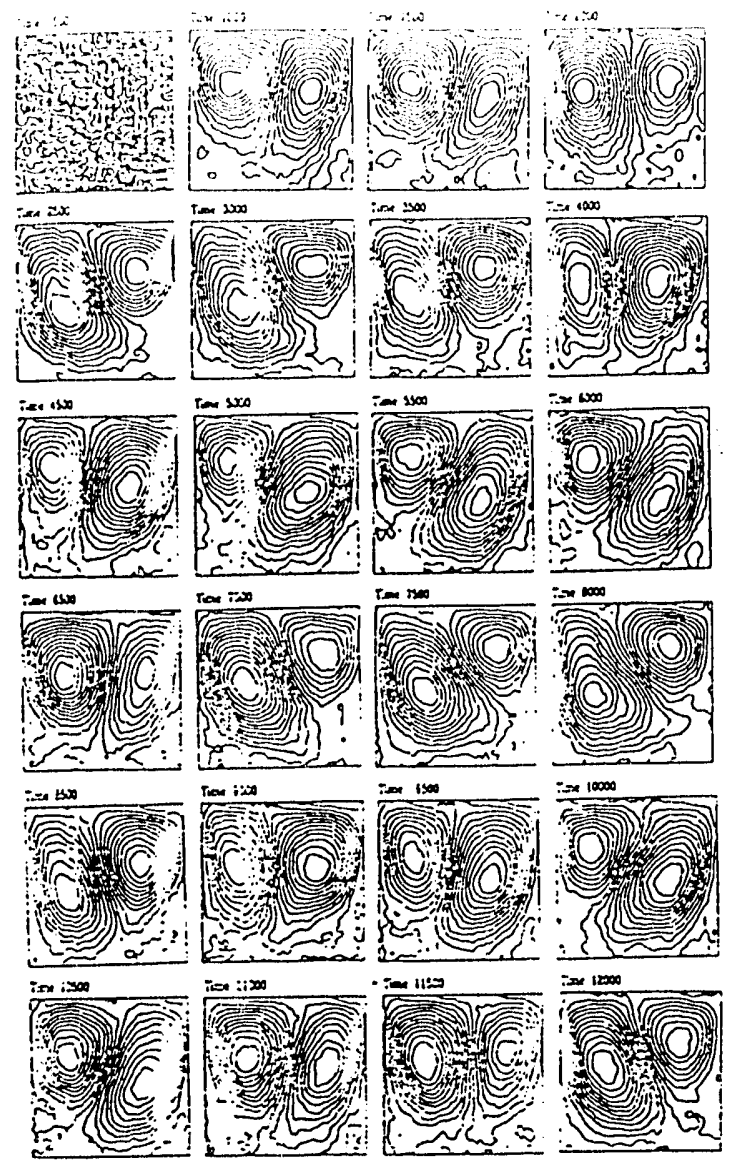

Figure 6. Rapaport's simulation of Rayleigh-Bénard flow. The time exposures [left] show vertical oscillations of the two vortices. The underlying fluid consists of 57,600 hard disks at about half the freezing density.

Figure 7. Goldhirsch, Pelz, and Orszag's simulation of Rayleigh-Bénard flow. Boundary collocation, with $32^{2}$ points, was used. This Figure [right] shows a four roll convective structure which recurs periodically between two mirror-image structures, each of which is dominated by a single diagonal roll.

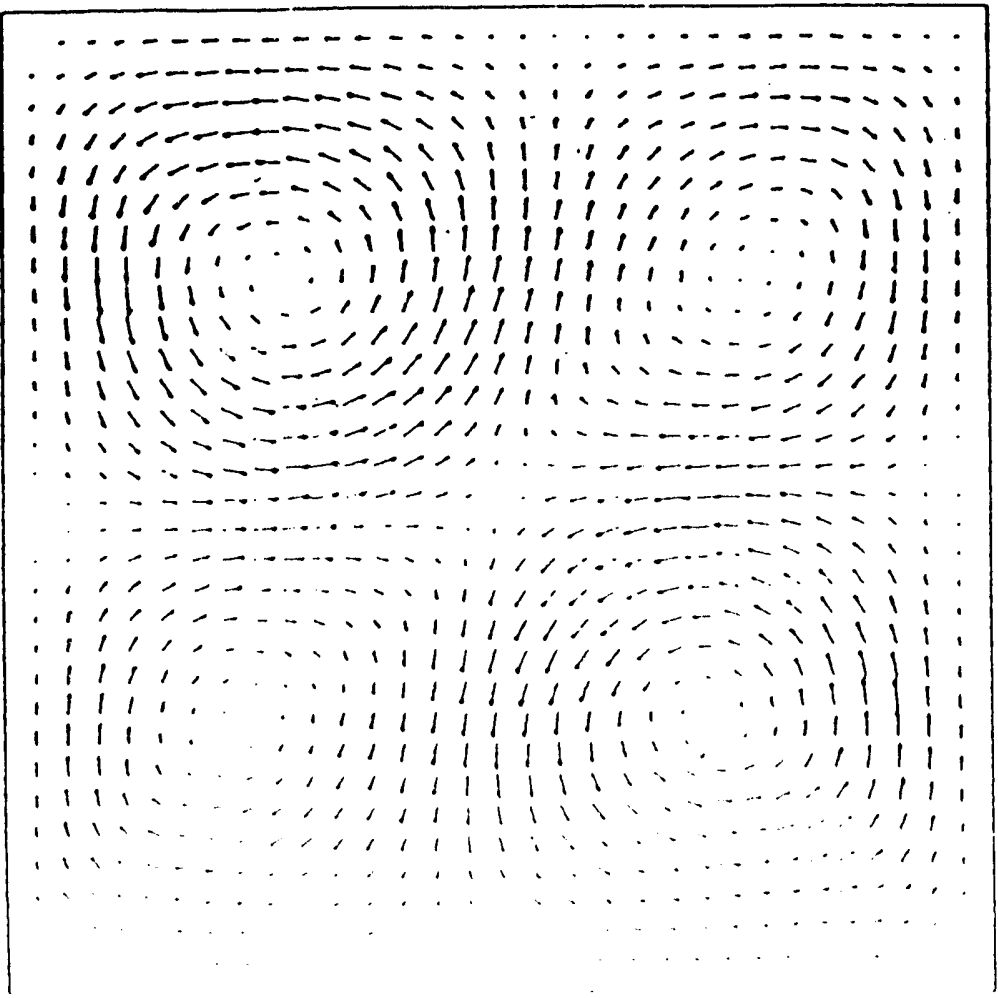




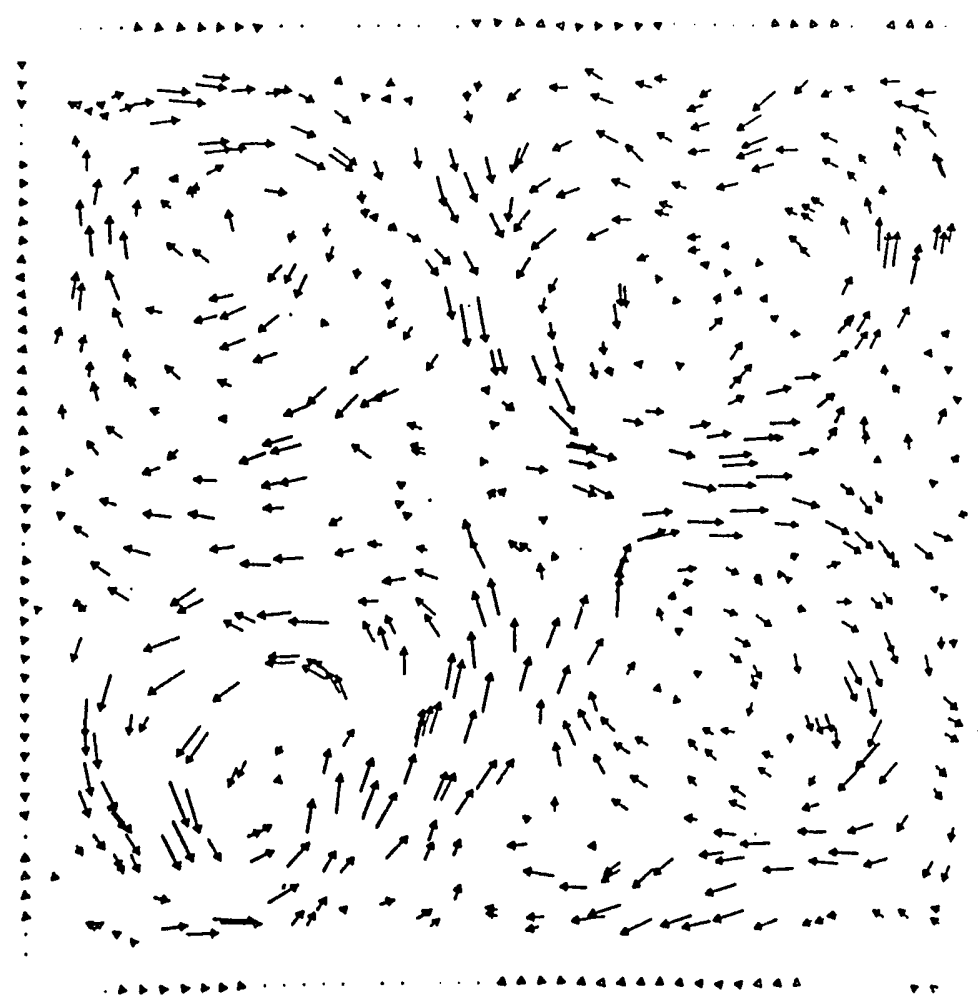

Figure 8. Smoothed-particle Hydrodynamic simulation of RayleighBénard flow for an ideal gas at unit mean density. The dimensionless analog of the Rayleigh number, $\mathrm{gh}^{3} /(\mathrm{vK})$, where $\mathrm{v}$ and $\mathrm{K}$ are the kinematic viscosity and thermal diffusivity, is $25 \times 10^{6} .200$ of the 776 smoothed particles are fixed, to define the four boundaries. The smoothed velocities $\{<v>\}$ of the individual particles are shown as arrows.
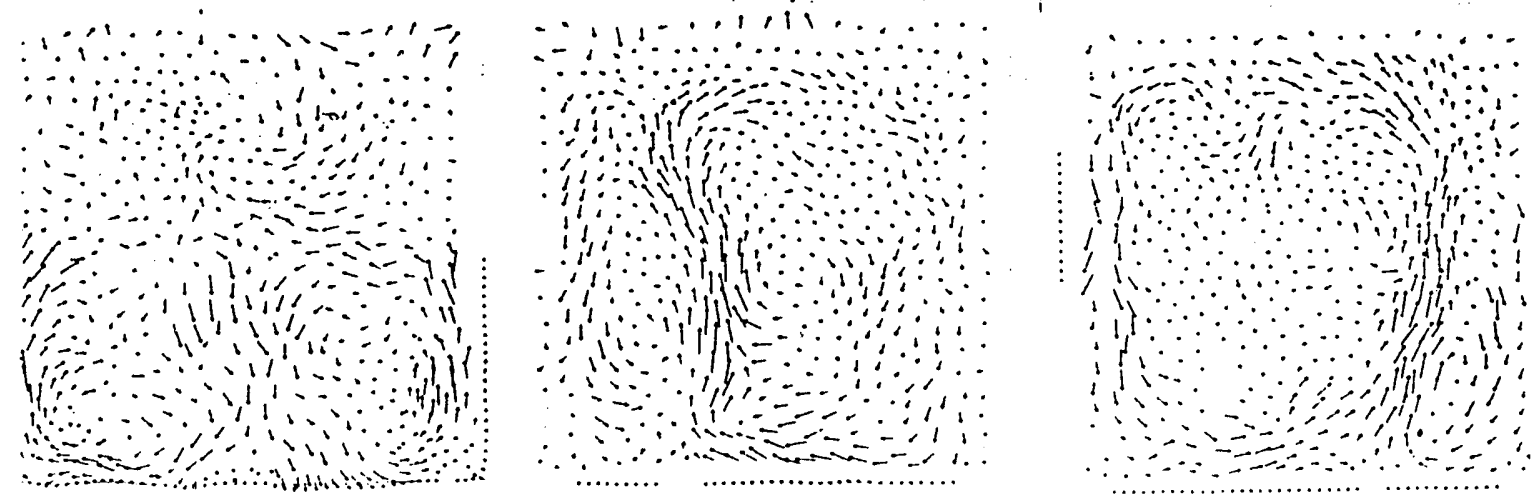

Figure 9. Smoothed-particle Hydrodynamic simulations of RayleighBénard flow. See Figure 7 . Here $\mathrm{gh}^{3} /(v \kappa)$ is near the apparent convection threshhold, $6 \times 10^{6}$. The snapshots are for three different simulations, with weighting function ranges of 2.0(left), 2.5(center), and 3.0(right). 
6. Surfaces, Failure, and Hybrid Models

Failure, whether of a liquid or a solid, involves the energy cost of bond breaking. New surfaces form through microscopic and chaotic irreversible processes. Despite the chaos, a detailed atomistic description of such a failure process is a straightforward application of Newton's motion equations. No special boundary conditions or constitutive assumptions are required. Figure 10 shows the breakup of a hot liquid drop.

Throughout any such failure process the forces on each atom follow from the same continuous force law. In the solid-phase deformation shown in Figures 2 and 3, our 373,248-atom crystal of indented silicon did not crack, even though solid silicon is normally a brittle material. The ductile behavior results because the strain energy, proportional to the solid's volume, does not exceed the surface energy necessary to nucleate a crack. Crack energy varies as area, so that large enough specimens do crack. The ductile small-scale behavior makes it possible to machine normally-brittle materials provided that sufficiently small tools (typically microscale single-point diamonds) are used.

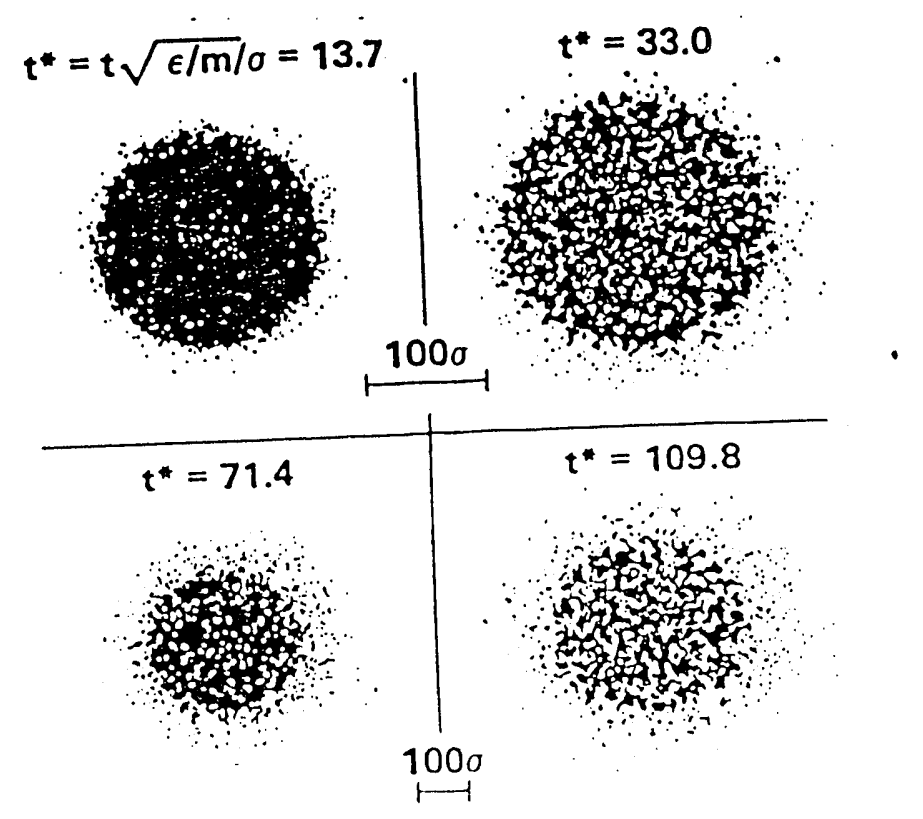

Figure 10. Fragmentation of a (two-dimensional) liquid drop of 14,491 liquid atoms, as described by Lennard-Jones' potential. From Blink's 1984 Ph. D. thesis work at the University of California at Davis/Livermore. 
With a failure strain of order $1 \%$, the elastic energy stored in a solid workpiece volume $\mathrm{V}$ is approximately $0.0001 \mathrm{BV}$, where $\mathrm{B}$ is the bulk modulus. The modulus itself is an energy density, of order $\varepsilon / \sigma^{3}$, where $\varepsilon$ and $\sigma$ describe the strength and range of a nearest-neighbor interaction. When $0.0001 \mathrm{~V} \varepsilon / \sigma^{3}$ exceeds the surface energy, $\left(\varepsilon / \sigma^{2}\right) \mathrm{V}^{2} / 3$, brittle fracture occurs. For fracture $\left(\mathrm{V} / \sigma^{3}\right)$ must be of order $10^{12}$, a bit bigger than the biggest feasible simulations. Thus a quantitative study of the limits of ductile machining, including fracture, requires a supporting substrate for the atomistic indented region in which to store the failure energy. The continuum substrate needs to join smoothly to the atomistic region, and to transmit phonons and heat, without excessive scattering at the boundary.

From the continuum perspective, failure is complicated by phase discontinuities and by surface tension. There is no problem in treating those parts of a solid remote from the indentor. For most of the workpiece material Hooke's Law should do. But whenever fracture occurs, an atomistic separation occurs, with a part of the stored energy of the solid localized on a fresh surface, and with the recoil of the fracture surfaces dependings sensitively on unstable multiple-valued regions of the phase diagram. It is therefore logical to combine the accurate atomistic description of failure, in the region of the indentor, with the more efficient continuum model for the remote substrate. Such a description can be facilitated by spanning the junction with a "hybrid" model combining atoms with Lagrangian zones. This is a promising research area.

We have already studied, in our 1992 Computers in Physics article, one approach to a hybrid inodel, filling some Lagrangian zones with atoms while treating others as continua. The impulses due to atomistic collisions with a zone wall (treated by constructing image particles on the other side of that wall) can then be summed up and used to accelerate those continuum nodes contiguous to that wall.

An aesthetically appealing method for joining the atomistic and continuum models is to use a particle description for them both. The enhanced continuum fluctuations in this approach resemble the thermal motion of an atomistic system. In the next Section we describe such an approach, in which the atomistic and continuum descriptions $t$ th use particles. It is to be expected that, in the absence of thermal motion, both descriptions provide exactly the same adiabatic response to accelerations. 
7. Simple Hybrid Model

The simplest hybrid atomistic+smoothed-particle description has a fixed weighting function $w(r)$, varying neither in time nor in space. Provided that the masses of the smoothed particles are the same as those of the atoms we then have a variety of ad hoc recipes which could be used to accelerate the atomic masses and the continuum nodes. The weighting function itself appears to be crucial to the success of the smoothed-particle approach. How should it be chosen? A choice based on reproducing the pair distribution function is a natural one. Thus the fundamental statistical mechanics of the correspondence between the atomistic and smoothed-particle distribution functions is well worth pursuing.

Even with an appropriate weight function selected, the hybrid dynamics is not entirely straightforward. In order to use the current information $\{r, v, e\}$ for the atoms and the nodes to calculate the time derivatives $\{\dot{r}, \dot{v}, \dot{e}\}$, stress, smoothed density and velocity, and the velocity gradient need to be estimated. In the mixed case this means estimating values for the tensors $\left(\sigma / \rho^{2}\right)$ and $\nabla v$ for an atomistic particle. This can be done adding the local contributions to the stress tensor and density in the neighborhood of the particle using the weight function w. Once this averaging has been completed, the pair contributions to the accelerations can be based on a coupling parameter $\lambda$ :

$$
a_{i j}=-(\lambda / m) \nabla \phi_{i j}+(1-\lambda) m \nabla_{i} w_{i j}\left[\left(\sigma / \rho^{2}\right)_{i}+\left(\sigma / \rho^{2}\right)_{j}\right] .
$$

It is natural to choose the values $\{0,1 / 2,1\}$ for the three possible types of $i j$ combinations. A successful hybridization of the two approaches must reproduce not only mechanical and thermal equilibria; it must also describe the linear transport of momentum and energy consistently.

For the atomistic part of these simulations we chose a very simple pair potential, with three vanishing derivatives at the cutoff, $r=1$ :

$$
\phi(r)=100\left(1-r^{2}\right)^{4} .
$$

In the smoothed-particle part of the simulations we use the constitutive model measured for this force law, including the transport coefficients $\eta$ and $\kappa$. For this purpose, a limited portion of the energy-density phase 
diagram was covered, using 100 particles. The specific heat and pressurevolume-energy constitutive equations could then be expressed as loworder polynomials in density and energy.

The smoothed particle idea has such flexibility that combinations of it with other forms of mechanics suffer from excessive richness.

Nevertheless, the promise of hybrid simulations demands an exploration of this uncharted but promising territory. At the time of this writing June, 1993) we have not completed hybrid simulations of shear flows and heat flows. I expect to describe such simulations in my talk in October.

Much still remains to be done in extending the range of applications for smoothed particle hydrodynamics. One particularly promising application of the smoothed-particle approach is the study of mesoscopic hydrodynamic fluctuations. The method is ideal for treating fluctuations on intermediate length and time scales.

\section{Summary}

With massively-parallel teraflop computers on the near horizon the transition from millions to billions of degrees of freedom is quite near. This development suggests connecting the microscopic models with more macroscopic approaches, so as to combine microscopic realism with macroscopic size in dealing with real problems.

\section{Acknowledgment}

I thank Professor Steinhauser for the opportunity to attend and to address this conference. Larry Cloutman was a helpful guide to smoothed-particle lore. Harald Posch provided some welcome assistance with the molecular dynamics. Brad Holian alerted me to the BeazleyLomdahl preprint listed in the references. I thank Drs. Beazley and Lomdahl for sending me an advance $\mathrm{CO}_{\mathrm{F} Y} \mathrm{y}$ of their work. The Lawrence Livermore National Laboratory and the University of California at Davis/Livermore both provided resources necessary to the completion of this work. Work performed at the Livermore Laboratory was carried out under the auspices of the United States Department of Energy, pursuant to Contract W-7504-Eng-48. 
Some General References:

Microscopic Molecular Dynamics:

See W. G. Hoover, Computational Statistical Mechanics (Elsevier, Amsterdam, 1991) and Microscopic Simulations of Complex Flows (M. Mareschal, editor), NASI Series B, Volume 236 (Plenum, New York, 1990) and the references below for an introduction to the literature. For a sample of recent timing results see D. M. Beazley and P.S. Lomdahl, "Message-Passing Multi-Cell Molecular Dynamics on the Connection Machine 5" (Parallel Computing, to appear).

Macroscopic Smoothed-Particle Hydrodynamics:

L. B. Lucy, "A Numerical Approach to the Testing of the Fission Hypothesis", Astronomical Journal 82, 1013 (1977).

J. J. Monaghan, "Smoothed Particle Hydrodynamics", Annual Review of Astronomy and Astrophysics 30, 543 (1992).

L. Hernquist, "Some Cautionary Remarks about Smoothed Particle Hydrodynamics", The Astrophysical Journal 404, 717 (1993).

W. G. Hoover, T. G. Pierce, J. Shugart, C. Stein, C. G. Hoover, and A. L. Edwards, "Molecular Dynamics, Smoothed-Particle Hydrodynamics, and Irreversibility", Computers \& Mathematics with Applications (to appear, 1994).

Microscopic and Macroscopic Simulations of Rayleigh-Bénard Instability:

Goldhirsch, R. B. Pelz, and S. A. Orszag, "Numerical Simulation of Thermal Convection in a Two-Dimensional Finite Box", Journal of Fluid Mechanics 199, 1 (1989).

D. C. Rapaport, "Molecular Dynamics Study of Rayleigh-Bénard Convection", Physical Review Letters 60, 2480 (1988) and "Temporal Periodicity in Microscopic Simulation of Rayleigh-Bénard Convection", in Microscopic Simulations of Complex Hydrodynamic Phenomena (M. Mareschal and B. L. Holian, editors), NASI Series B, Volume 292 (Plenum, New York, 1992).

Microscopic Simulations of Silicon Indentation:

W. G. Hoover, A. J. De Groot, and C. G. Hoover, “Massively Parallel Computer Simulation of Plane-Strain Elastic-Plastic Flow via Nonequilibrium Molecular Dynamics and Lagrangian Continuum Mechanics", Computers in Physics 6, 155 \& cover (1992).

J. S. Kallman, W. G. Hoover, C. G. Hoover, A. G. DeGroot, S. M. Lee, and F. Wooten, "Molecular Dynamics of Silicon Indentation", Physical Review B 47, 7705 (1993). 

